\title{
Bioanalysis
}

\section{Sweat as a clinical sample: what is done and what should be done}

\begin{abstract}
"The clinical importance of sweat has traditionally been limited to the determination of chloride for the diagnosis of cystic fibrosis and incipient determination of drugs."
\end{abstract}

First draft submitted: 30 September 2015; Accepted for publication: 21 October 2015; Published online: 10 December 2015

Keywords: sweat metabolomics • sweat normalization • sweat sample preparation • sweat samplers • sweat xenometabolomics

"The role of sweat in clinical analysis is poor, but this sample deserves a prominent place that can be achieved after development of appropriate research."

Sweat is known for being a clear, hypotonic biofluid produced by eccrine and procrine glands located in the epidermis, with a slightly acidic $\mathrm{pH}$ (between 4.0 and 6.8), and composed mainly by water (99\%), containing the so-called electrolytes (e.g., sodium, chloride and potassium), urea, pyruvate and lactate; but also proteins, peptides, amines, amino acids and metal ions in smaller concentrations, together with inhibitors, antigens, antibodies and a variety of xenobiotics such as drugs, cosmetics and ethanol [1]. The clinical importance of sweat has traditionally been limited to the determination of chloride for the diagnosis of cystic fibrosis $(\mathrm{CF})$ and incipient determination of drugs [1]. The present spread of 'omics' disciplines, and particularly of metabolomics as the youngest of the big 'omics,' has open a fan of possibilities to the use of sweat as clinical sample. Except for the case of some high molecular weight proteins, which reach sweat by different intracellular storages in particular situations $[2,3]$, most sweat components are small molecules resulting from metabolic pathways; therefore, their study pertains to the metabolomics field, the omics of small molecules typically (<1000 Da or $<1500 \mathrm{Da})$.

The present advantages of sweat as clini- cal sample that push a growing research are: its no invasive sampling, crucial in advanced states of some diseases and that makes unnecessary health personnel care; its simple but varied composition that can be the basis for its clinical interest for diagnostic. On the other hand, some present shortcomings are: the low secreted volume, the variable secretion among individuals and the lack of optimized analytical protocols, which are mainly necessary in metabolomics. Therefore, the main aspects to which immediate attention should be paid with the dual purpose of increasing the advantages and overcoming shortcomings should encompass all steps of the analytical process are as follows.

\section{Sweat sampling}

The diagnostic of some diseases such as CF promoted in the past the improvement of old sweat samplers such as occlusive bandages, giving place to present commercial Macroduct and Megaduct devices [4]. They alleviated shortcomings such as the difficulty to produce enough sweat for analysis, sample evaporation, need for a trained staff and errors in the results owing to the presence of pilocarpine; therefore, more precise the determination of chloride for CF diagnostic were then possible. Nevertheless, present more complex uses of sweat - mainly when involved in omics analyses - make mandatory the design of new samplers with total overcoming of present shortcomings and

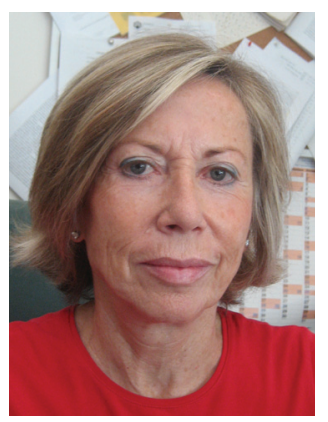

Maria Dolores Luque de Castro

Department of Analytical Chemistry, Annex Marie Curie Building, Campus of Rabanales, University of Córdoba, Córdoba, Spain

and

ceiA3 Agroalimentary Excellence Campus, University of Córdoba, Córdoba, Spain

and

Maimónides Institute of Biomedical Research (IMIBIC), Reina Sofía University Hospital, Córdoba, Spain qa1lucam@uco.es 
even offering new advantages. In dealing with sweat sampling for drug abuse monitoring, patches provide a long-term continuous monitoring of drug exposure [4]. The recent hydrogel micropatches for sampling and profiling skin metabolites are an example of the research in this area [5]. Also, the necessity for different devices for sweat sampling may result from more in depth studies on mapping different body zones as the irreproducibility of the present samplers (designed for sampling in arms) made imprecise the differences in sweat composition depending on the body area [6].

\section{Normalization of the sampled volume}

A pending goal in the use of sweat as clinical sample is normalization as the protocols assayed so far have not provided successful results. Thus, Appenzeller et al. [7] used the concentration of sodium and potassium as an internal reference to normalize the volume of sweat collected by patch samplers. The authors measured the target metabolites by capillary zone electrophoresis-diode-array detector for indirect detection at $214 \mathrm{~nm}$, and concluded that $\mathrm{K}^{+}$cannot be used for normalization owing to the high interindividual variability, while $\mathrm{Na}^{+}$displayed very low variability within groups, making it suitable for normalization. Nevertheless, the authors did not assayed the normalization system in sweat from CF patients, who alter their sodium concentration. More research on this analytical aspect is mandatory that should be developed with more complex instrumentation that provides more information on different parameters to be jointly treated.

\section{"A pending goal in the use of sweat as clinical sample is normalization as the protocols assayed so far have not provided successful results."}

\section{Sample preparation}

Sweat is a relatively simple sample that does not require preparation for CF diagnostic; while for other analyses it is mandatory treatment to lead the target analytes to the appropriate state for measurement, which depends on both the analytes and the analytical equipment. This is the case with drugs analysis [1] and in the so far scant applications in the genomics [8] and proteomics fields [9]. The recent use of sweat as sample to search for metabolomics biomarkers [10] or quantitative analysis of amino acids [11] has shown as unnecessary sample cleanup (using SpinColumn SPE cartridges because the low sample volume). Sample dilution demonstrated to be enough to overcome interferents and avoid derivatization reactions when LC-MS is used instead of an automatic amino acids analyzer [12]. More in depth research is necessary to propose other sample preparation steps that can be required particularly in targeted metabolomics analysis.

\section{Sweat analysis}

The complexity of equipment for sweat analysis depends on the target analyte(s).

In dealing with the determination of a single compound, as is the case for chloride for CF diagnostic, there are a number of dedicated instruments - usually simple commercial devices - based mainly on potentiometry, but also others based on osmolarity, conductivity or coulombimetric measurements [13]. Example of the interest in improving CF diagnosis is the recent CF quantum ${ }^{\circledR}$ sweat test [14]. Mention apart among single analytes determined in sweat deserves $\mathrm{pH}$. This parameter can be correlated to sodium concentration and sweat rate in order to indicate to users the proper time to hydrate during physical exercise and avoid the risk of muscle cramps; and also changes in the $\mathrm{pH}$ of the skin play a role in the pathogenesis of skin diseases, such as dermatitis and acne [15]. These known aspects of sweat-pH monitoring have promoted the development of a smartphone based accessory and method for the rapid colorimetric detection of $\mathrm{pH}$ in sweat [16], and also an autonomous, robust and wearable microfluidic platform capable of performing online analysis of $\mathrm{pH}$ in sweat [17]. These and similar devices can help in searching for other relationships between sweat $\mathrm{pH}$ and individual healthy/ill states.

Sweat is also an excellent sample for drug analysis as the whole human body produces between 300 and $700 \mathrm{ml} /$ day of sweat. In the context of the absorption/distribution/metabolism/excretion cycle of drugs, a small but sufficient fraction of a drug is excreted in sweat; therefore, drug can be tested in this biofluid [1] with several advantages over blood or urine such as less opportunity for sample adulteration, longer time-detection windows in some cases and noninvasiveness as compared with blood [1]; however, analyses of the drug in sweat provided a time window detection of up to a week or more after administration [1]. Other advantage of sweat as clinical sample is that drugs with basic nature can accumulate in sweat from blood due to the difference of $\mathrm{pH}$ between the two matrices [1]. An aspect to be taken into account in future research is that most of the authors working on this field determine the target drug (at LOQ as low as $0.4 \mathrm{ng}$ drug/sample), but not its metabolites [18]. Monitoring the precursor and metabolites in individuals under different conditions could provide information on potential different metabolic pathways closely related with the healthy/ill state of the individual.

Along with essential metals, sweat is also a known excretory route for xenometals; therefore, their analysis 
with subsequent interpretation of comparative results are a xenometabolomics matter. In addition, depending on the oxidation state of the target metal, the research belongs to metabolomics subdisciplines such as ionomics or metallomics. Metals xenometabolites can be no toxic, as is the case with $\mathrm{Ni}$, or toxic as $\mathrm{Cd}$, $\mathrm{Pb}, \mathrm{Hg}$ and As; all them can enter the organism as metals or salts and, when absorbed through the skin, they are first solubilized by sweat. Therefore, blood or urine might not reflect properly dermal absorption, which is better expressed in sweat. In addition, optimization of the potential of sweating as a therapeutic excretory mechanism merits further research, as does research into homeostasis of no common metals, and conditioning or adaptation to regular sweating by athletes to be matched with studies of excretion of trace elements.

Nevertheless, it is the search for metabolomics biomarkers of key diseases as lung cancer [19] the area of outstanding interest, as demonstrated by the changes in the excreted metabolites with different advancement of the target disease. The growing development of metabolomics data treatment and metabolomics

\section{References}

1 Mena-Bravo A, Luque de Castro MD. Sweat: a sample with limited present applications and promising future in metabolomics. J. Pharm. Biomed. Anal. 90, 139-147 (2014).

2 Sato K, Kang WH, Saga K, Sato KT. Biology of sweat glands and their disorders. I. Normal sweat gland function. J. Am. Acad. Dermatol. 20, 537-563 (1989).

3 Raiszadeh MM, Ross MM, Russo PS, Schaepper MA et al. Proteomic analysis of eccrine sweat: implications for the discovery of schizophrenia biomarker proteins. J. Proteome Res. 11, 2127-2139 (2012).

4 Ely MR, Ely BR, Chinevere TD et al. Evaluation of the megaduct sweat collector for mineral analysis. Physiol. Meas. 33, 385-394 (2012).

5 Dutkiewicz EP, Lin JD, Tseng TW et al. Hydrogel micropatches for sampling and profiling skin metabolites. Anal. Chem. 86, 2337-2344 (2014).

6 Kutyshenko1 VP, Molchanov M, Beskaravayny P et al. Analyzing and mapping sweat metabolomics by highresolution NMR spectroscopy. PLoS ONE 6(12), e28824 (2011).

7 Appenzeller BMR, Schummer C, Boura-Rodrigues $S$, Wennig R. Determination of the volume of sweat accumulated in a sweat-patch using sodium and potassium as internal reference. J. Chromatogr. B Analyt. Technol. Biomed Life Sci. 852, 333-337 (2007).

8 Patterson MJ, Galloway SDR, Nimmo MA. Variations in regional sweat com-position in normal human males. Exp. Physiol. 85, 869-875(2000). databases opens new possibilities to the information provided by cutting-edge analytical instrumentation, excellent tools for the small volumes characteristic of sweat samples.

\section{Conclusion}

Further research is mandatory to demonstrate and take profit from the wide potential of sweat as clinical sample. Metabolomics is the field in which the most fruitful results are foreseeable.

\section{Financial \& competing interests disclosure}

Junta de Andalucía and FEDER programme are gratefully acknowledged for financial support (Project 'Optimización y aplicación de plataformas metabolómicas de análisis de biofluidos no invasivos para la búsqueda de biomarcadores de diagnóstico precoz de cáncer de pulmón, FQM-1602'). The author has no other relevant affiliations or financial involvement with any organization or entity with a financial interest in or financial conflict with the subject matter or materials discussed in the manuscript apart from those disclosed.

No writing assistance was utilized in the production of this manuscript.
9

Porter D, Weremowicz S, Chine K et al. A neural survival factor is a candidate oncogene in breast cancer. Proc. Natl Acad. Sci. USA 100, 10931-10936 (2003).

10 Mark H, Harding CR. Amino acid composition, including key derivatives of eccrine sweat: potential biomarkers of certain atopic skin conditions. Int. J. Cosmetic Sci. 35, 163-168 (2013).

11 Calderón-Santiago M, Priego-Capote F, Jurado-Gámez B, Luque de Castro MD. Optimization study for metabolomics analysis of human sweat by liquid chromatography-tandem mass spectrometry in high-resolution mode. J. Chromatogr. A 1333, 70-78 (2014).

12 Delgado-Povedano MM, Calderón-Santiago M, PriegoCapote F, Luque de Castro MD. Study of sample preparation for quantitative analysis of amino acids in human sweat by liquid chromatography-tandem mass spectrometry. Talanta 146, 310-317 (2015).

13 Domingos MT, Rodrigues Magdalena NI, Nunes Lima M et al. Sweat conductivity and coulometric quantitative test in neonatal cystic fibrosis screening. Jornal de Pediatria doi: 10.1016/j.jped.2015.03.003 (2015) (Epub ahead of print).

14 Rock MJ, Makholm L, Eickhoff J. A new method of sweat testing: the CF Quantum ${ }^{\circledR}$ sweat test. J. Cystic Fibrosis 13, 520-527 (2014).

15 Schmid-Wendtner MH, Korting HC. The $\mathrm{pH}$ of the skin surface and its impact on the barrier function. Skin Pharmacol. Physiol. 19, 296-302 (2006).

16 Oncescu V, O’Dell D, Erickson D. Smartphone based health accessory for colorimetric detection of biomarkers in sweat and saliva. Lab Chip 13, 3232-3240 (2013). 
17 Curto VF, Coyle S, Byrne R, Angelov N et al. Concept and development of an autonomous wearable micro-fluidic platform for real time $\mathrm{pH}$ sweat analysis. Sensors Actuators $B$ 175, 263-270 (2012).

18 Huestis MA, Scheidweiler KB, Saito T, Fortner N. Excretion of $\Delta 9$-tetrahydrocannabinol in sweat. Forensic Sci. Int. 74(2/3), 173-177 (2008).
19 Calderón-Santiago M, Priego-Capote F, Turck N et al. Human sweat metabolomics for lung cancer screening. Anal. Bioanal. Chem. 407, 5381-5392 (2015). 